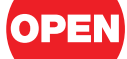

SUBJECT AREAS:

ANIMAL BEHAVIOUR

BEHAVIOUR

BIOLOGICAL MODELS

SENSORY SYSTEMS

Received

30 January 2012

Accepted

23 March 2012

Published

17 April 2012

Correspondence and requests for materials should be addressed to M.K. (mknaden@ice. mpg.de)

* authors share senior authorship.

\section{A high-throughput behavioral paradigm for Drosophila olfaction - The Flywalk}

\author{
Kathrin Steck', Daniel Veit' , Ronald Grandy' , Sergi Bermúdez i Badia'², Zenon Mathews ${ }^{3}$, Paul Verschure ${ }^{3}$, \\ Bill S. Hansson ${ }^{1 *} \&$ Markus Knaden ${ }^{1 *}$
}

\begin{abstract}
${ }^{1}$ Max Planck Institute for Chemical Ecology, Jena, Germany, ${ }^{2}$ Madeira Interactive Technologies Institute, University of Madeira Carnegie Mellon | Portugal - Madeira Tecnopolo, 9020-105 Funchal, Portugal, ${ }^{3}$ Laboratory of Synthetic Perceptive, Emotive and Cognitive Systems, ICREA-UPF, Barcelona, Spain.
\end{abstract}

How can odor-guided behavior of numerous individual Drosophila be assessed automatically with high temporal resolution? For this purpose we introduce the automatic integrated tracking and odor-delivery system Flywalk. In fifteen aligned small wind tunnels individual flies are exposed to repeated odor pulses, well defined in concentration and timing. The flies' positions are visually tracked, which allows quantification of the odor-evoked walking behavior with high temporal resolution of up to $100 \mathrm{~ms}$. As a demonstration of Flywalk we show that the flies' behavior is odorant-specific; attractive odors elicit directed upwind movements, while repellent odors evoke decreased activity, followed by downwind movements. These changes in behavior differ between sexes. Furthermore our findings show that flies can evaluate the sex of a conspecific and males can determine a female's mating status based on olfactory cues. Consequently, Flywalk allows automatic screening of individual flies for their olfactory preference and sensitivity.

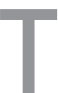

he vinegar fly Drosophila melanogaster has become a model organism for olfaction mainly due to the impressive genetic toolbox available, which enables us to manipulate the neuronal architecture of the olfactory system. These manipulations help to understand how odorants are detected by the peripheral olfactory sensory system ${ }^{1,2}$ and how information is further processed in the first olfactory processing centre, the antennal lobes ${ }^{3-6}$. While neurophysiological characteristics can be precisely quantified (e.g. spike frequency of a specific neuron or activity changes within a set of glomeruli of the antennal lobe), quantification of odorantinduced behavioral changes in Drosophila still leaves much to be desired. Up to now the effect of minute changes deriving from genetic manipulations of the fly's sensory system were discovered rather by elaborate neurophysiological measurements than in quantitative behavioral assays. In order to compensate for the high variability of behavioral data an ideal bioassay would require the synchronized observation of many individual flies and their responses to repeated stimuli well-defined in quality, quantity, and stimulus on- and off-set. In addition, to correlate behavioral and physiological data, stimulus presentation during behavioral and physiological experiments should be similar, i.e. short pulses followed by prolonged pauses of clean air. At present olfactory driven fly behavior is mostly investigated in mass experiments, as trap assays ${ }^{7}$ and $\mathrm{T}$-mazes ${ }^{8}$, in which large groups of flies are asked to choose between one odorant and a control. Both bioassays use continuous olfactory stimulation rather than odor pulses, which precludes any evaluation of olfactory response latencies. Furthermore, continuous olfactory stimulation might cause adaptation of the flies' sensory system to an odor, and thus make any evaluation of olfactory sensitivity difficult. Moreover, this approach is ecologically invalid as most odor driven behavior occurs in turbulent plumes. Finally, olfactory behavior in mass experiments is quantified by the fraction of responding flies. Hence, these experiments reveal the olfactory preferences of the plurality and not of individual flies.

Experiments with individual flies have been conducted in 2- and 4-way olfactometers ${ }^{5,9,10}$, in arenas with stationary air and flowing air conditions ${ }^{11}$, in wind tunnels ${ }^{12}$, and in a tethered-fly paradigm ${ }^{13-15}$. Notably, the 2 -way olfactometer experiment ${ }^{10}$ allows testing numerous individual flies simultaneously while investigation of latency and strength of the flies' olfactory responses is disregarded. By using two different arena setups Keller and Vosshall ${ }^{11}$ screened behavior of individual flies with 73 different odorants. Measuring the activity change of the flies after stimulus arrival allowed identifying odorants that were detected by the flies. However, the hedonic valence of the odorants could not be assessed. Contrary to the other experiments mentioned, the wind tunnel ${ }^{12}$ and the tethered-fly experiment ${ }^{13-15}$ allow for the assessment of response latency and sensitivity. However, these setups are designed for single flies and in order to gain meaningful sample sizes the experiments need to be repeated many times. 
To remedy the shortcomings of presently used vinegar fly bioassays, we developed the Flywalk, a novel high-throughput paradigm for studying odorant-evoked behavior in walking Drosophila. The communication between the tracking system and the odor-delivery system allows using the Flywalk as an open-loop paradigm as described in the present work, with information being sent from the odor-delivery system to the tracking system. In addition the Flywalk can be programmed to meet demands of a closed-loop system, where the behavior of the flies triggers the onset of the odordelivery system (i.e. whenever all flies, or a given percentage of the flies are motionless, an odor stimulus is delivered). In the current version of the system we track 15 freely walking flies simultaneously in parallel small-sized wind tunnels (length, $18 \mathrm{~cm}$; inner diameter, $0.8 \mathrm{~cm}$; constant air flow of $18 \mathrm{~cm} / \mathrm{s}$ ) (Fig. 1). Each of the flies is exposed to identical odorant pulses, well-defined in quality, quantity and stimulus duration (for characterization of the odor stimulus see Fig. 2 and Supplementary Information online, for description of the stimulus device $\mathrm{see}^{16}$ ). Communication between the stimulus delivery system and the automated tracking system allows calculation of the exact time of the encounter between the stimulus and fly (i.e. the meeting time, based on wind speed, stimulus onset and position of the fly within the tube). This calculation enables detailed analysis of the behavior of individual flies before, during and after odor stimulation. A total of eight different odorant stimuli can be presented repeatedly and the sequence of these is randomized with a stimulus interval of $90 \mathrm{sec}$. This representative protocol results in an experimental session that lasts for eight hours during which all 15 flies are exposed in parallel to 40 stimulations per odorant, and allows for an analysis of the response of each individual fly as well as the summed responses of a group of flies to an odor, further referred to as the sum response. The analysis presented here of the responses of individual flies and of sum responses of numerous flies revealed odorant- and concentration-specific changes in the flies' undirected activity and directed up- or downwind movements. Hence, the Flywalk bioassay for the first time allows screening of large numbers of individual flies for their odorant preferences, olfactory sensitivities, and response latencies in an ecologically valid way.

As a demonstration of the properties of Flywalk we show that:

- fly responses are odorant specific, i.e. stimulation with different odors results in different patterns of directed up- or downwind movements (i.e. attraction or repellence) and/or changes in undirected activity.

- odor responses are sex specific, i.e. responses of female and male flies differ in strength and duration to food-related odors and to the Drosophila pheromone cis-vaccenyl acetate, while both sexes respond similarly to repellent odorants.

- Orco mutant flies (lacking most functional olfactory receptors) respond to some odorants with a change in activity but show no directed movements, i.e. attraction or repellence.

- volatile cues are sufficient for male flies to evaluate sex and mating status of female conspecifics.

\section{Results}

A representative experimental session lasted for up to eight hours, with flies being repeatedly exposed to short odorant pulses (for detailed description of odor delivery, see Methods section). During the time between the pulses, the flies could move freely and redistribute. The point in time when an odor pulse met a fly (meeting time) could be determined based on the combined knowledge of

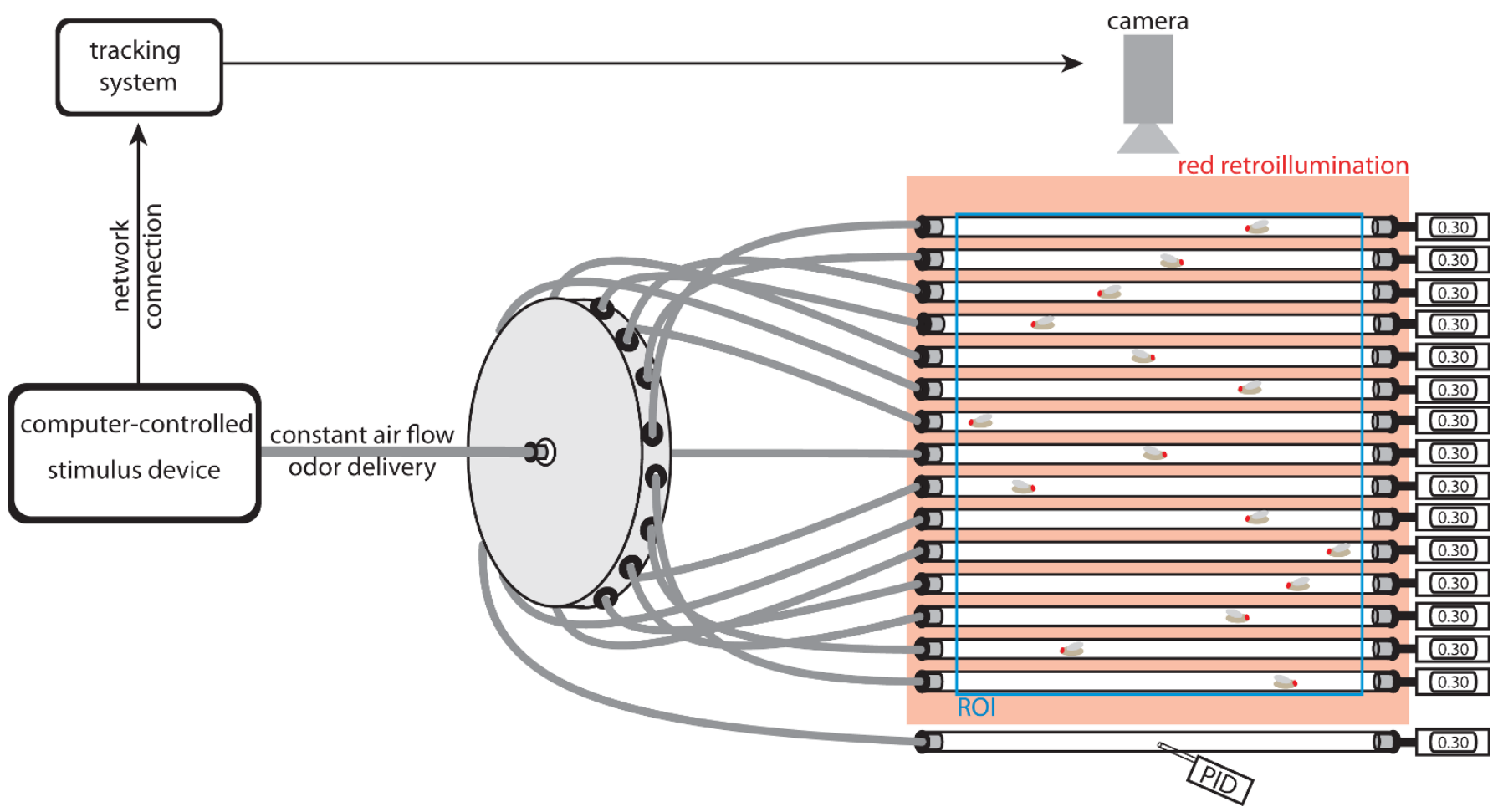

Figure 1 Experimental setup. 15 flies are individually exposed within glass tubes to a continuous air flow (speed, $18 \mathrm{~cm} / \mathrm{s}$; temperature, $25^{\circ} \mathrm{C}$; humidity, $70 \%$ ) and are automatically tracked. A computer-controlled stimulus system produces olfactory stimuli, which are transported by the air flow via a splitup board to the flies. The stimulus system communicates on- and off-set to the tracking system. Digital flow meters after each glass tube guarantee that airflow and, hence, stimulus movement are controlled and identical in all tubes. Based on position of the flies within the tubes and knowledge about stimulus timing and speed, the tracking system calculates each fly's responses to up to eight different olfactory stimuli with a temporal resolution of $100 \mathrm{~ms}$. A photo ionization detector (PID) plugged into an additional tube checks reliability of the stimulus device. Red rectangle, retroillumination $(>630 \mathrm{~nm})$ provided by electroluminescent foil to preclude visual cues. Flies positioned close to the tube ends, i.e. out of region of interest (ROI, open blue rectangle), are not tracked. 
stimulus onset, wind speed, and the actual position of each fly. (For a detailed analysis of stimulus characteristics and timing by means of measurements with a photo ionization detector and electroantennogram recordings within the tubes see Fig. 2 and Supplementary Information online). Based on the recorded actual positions of the flies and with the help of a MATLAB routine (The Mathworks, Natick, USA) the walking speed was calculated between $3 \mathrm{~s}$ before and $7 \mathrm{~s}$ after the meeting time (Fig. 3a).

Flies typically remained motionless or moved randomly during the pre-odor period (Fig. 3a). Also, flies did not change their movements upon meeting with pulses of the solvent control (Fig. 3a, mineral oil). However, after meeting pulses of ethyl acetate, a known attractant, flies exhibited increased upwind speed (blue squares in Fig. 3a). On the other hand, pulses of benzaldehyde, which has been described as a repellent ${ }^{17}$, evoked mainly downwind movements (red squares in Fig. 3a). These responses to the stimuli were stable during the whole experimental session (for an analysis of response stability of 30 flies see also Supplementary Fig. S4 online). Furthermore, the response to ethyl acetate was dose-dependent, with the strength of responses correlating well with stimulus concentration (Supplementary Fig. S7 online). As individual flies were stimulated repeatedly with each odorant, we could also test whether or not each fly was significantly attracted or repelled by it (Fig. 3b and Supplementary Fig. S5 online).

In addition to ethyl acetate and benzaldehyde, we tracked responses to pulses of balsamic vinegar and to cis-vaccenyl acetate (cVA, a pheromone involved in aggregation, mating, and aggression $\left.^{18-21}\right)$. We recorded responses of 30 female and 30 male wild type flies. For each individual fly we calculated the upwind speed and the undirected activity (i.e. whether the fly was moving or not, independent from the heading direction and walking speed) before, during and after encountering the stimulus. Based on repeated stimulations we calculated the median responses (i.e. the median walking speed and the activity) of each fly to each odor (for an example see single fly in Fig. 3a, for additional odorants see Supplementary Fig. S5 online).

Wild type flies of both sexes display a similar response repertoire to all odors regarding both undirected activity and directed movements (Fig. 4). However, sex-specific differences were found in strength and duration of the responses to food-related odors (balsamic vinegar and ethyl acetate, Fig. $4 \mathrm{~b}$ and c) and cVA (Fig. 4e). Female flies accelerated their upwind movements significantly more and longer after stimulation with food-related odors than male flies. Male flies on the other hand, responded stronger and longer lasting to cVA than female flies. As upwind movements would bring a fly closer to an odor source, we interpret odorants that provoke upwind movements as attractive. While all food-related odors and cVA provoked directed upwind runs 200-300 ms after meeting time, benzaldehyde resulted in a non-sex-specific, 500-ms lasting decrease in activity (from now on called "freezing") that was followed by a directed downwind movement (Fig. 4d). Freezing also occurred when flies were tested with 1-octen-3-ol (Supplementary Fig. S6 online), another odor that has been described to repel Drosophila ${ }^{22}$. We interpret the freezing and downwind movements as avoidance behavior.

To reconfirm the findings that the responses we observed are indeed olfactory guided we performed experiments with Orco mutant flies. These flies are lacking most olfactory receptors but are equipped with functional ionotropic chemical receptors that have been described to detect mainly acids and amines ${ }^{6}$. Orco $-/-$ flies were neither attracted nor repelled by any of the odors (Fig. 4, for additional odorants see Supplementary Fig. S6 online). However, activity patterns of Orco $-/-$ flies changed significantly when they were exposed to balsamic vinegar and benzaldehyde (Fig. $4 \mathrm{~b}$ and d). Orco $-/$ - flies exhibited prolonged freezing behavior after encounters with balsamic vinegar pulses (Fig. 4b). Benzaldehyde on the a
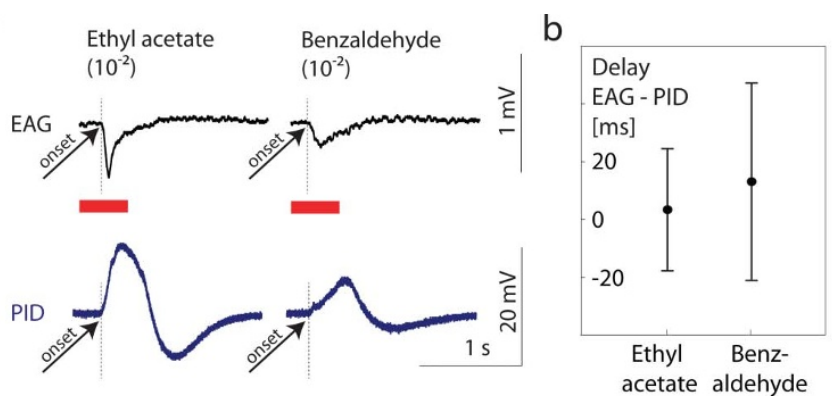

C

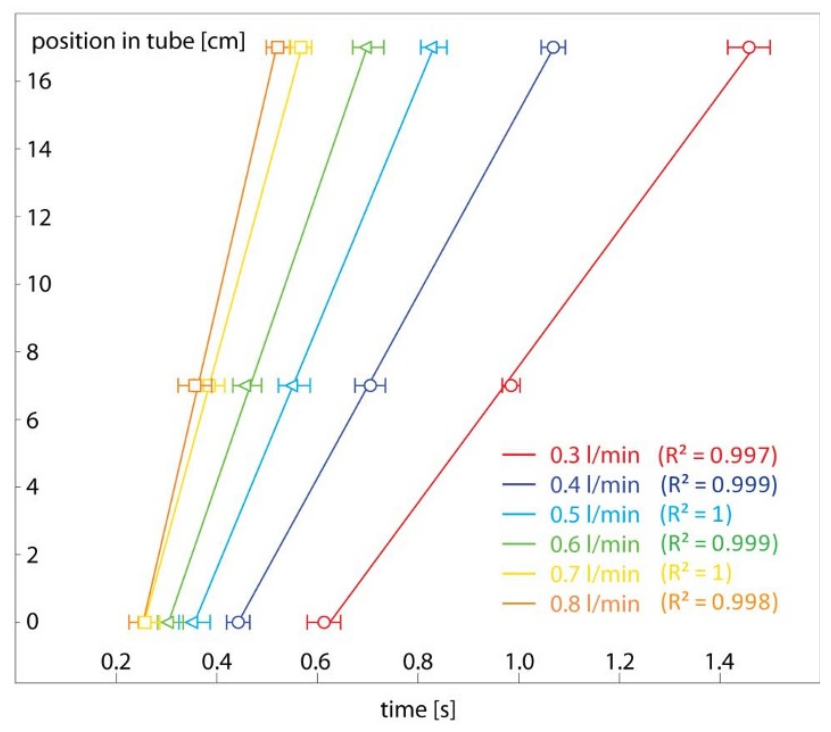

d

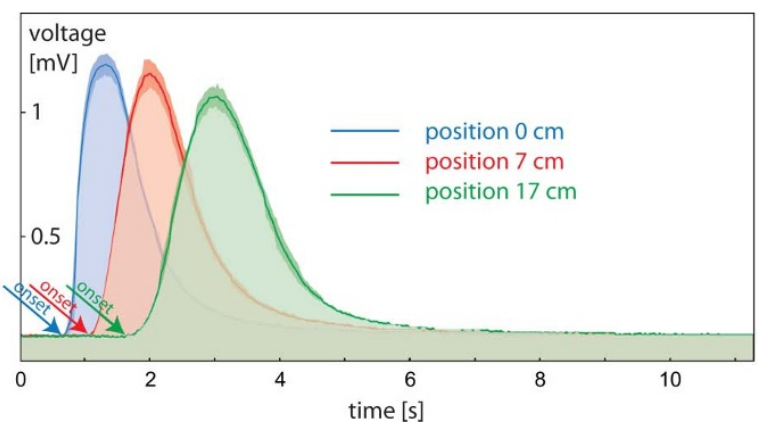

Figure $2 \mid$ Stimulus characterization. (a) Simultaneous PID and EAG recordings of odor pulses.We simultaneously recorded PID and EAG signals at different positions in the tube $(5 \mathrm{~cm}, 10 \mathrm{~cm}$ and $15 \mathrm{~cm})$ to $500 \mathrm{~ms}$ pulses of ethyl acetate and benzaldehyde ( $10^{-2}$ dilution). Both signals were recorded simultaneously using a 4-channel USB acquisition controller, type IDAC-4 (Ockenfels SYNTECH GmbH, Germany). Arrow heads depicts onset of responses. Please note that the Syntech software processes the analogue input of the PID signal with the same filters as used for EAG processing. Therefore, the course of the PID signal does not reflect the real but a processed course over the whole time elapse. However, it informs about the onset of the signal (arrow head). (b) Delay between time of onset in EAG and PID recordings after stimulation with ethyl acetate and benzaldehyde $(\mathrm{n}=10)$. We found no systematic delay between both methods applied $(n=10)$. (c) Calibration for speed of stimulus movement within the tubes $(n=10)$. We conducted PID measurements at three positions in the tube for five different air flows. Linear correlation between time and pulse position within the tube ensures that the stimulus is moving with a constant and predictable speed. (d) Shape of pulses depending on position in tube ( $\mathrm{n}=5$ for each measurement). Solid line, median; shaded area, interquartile range. 

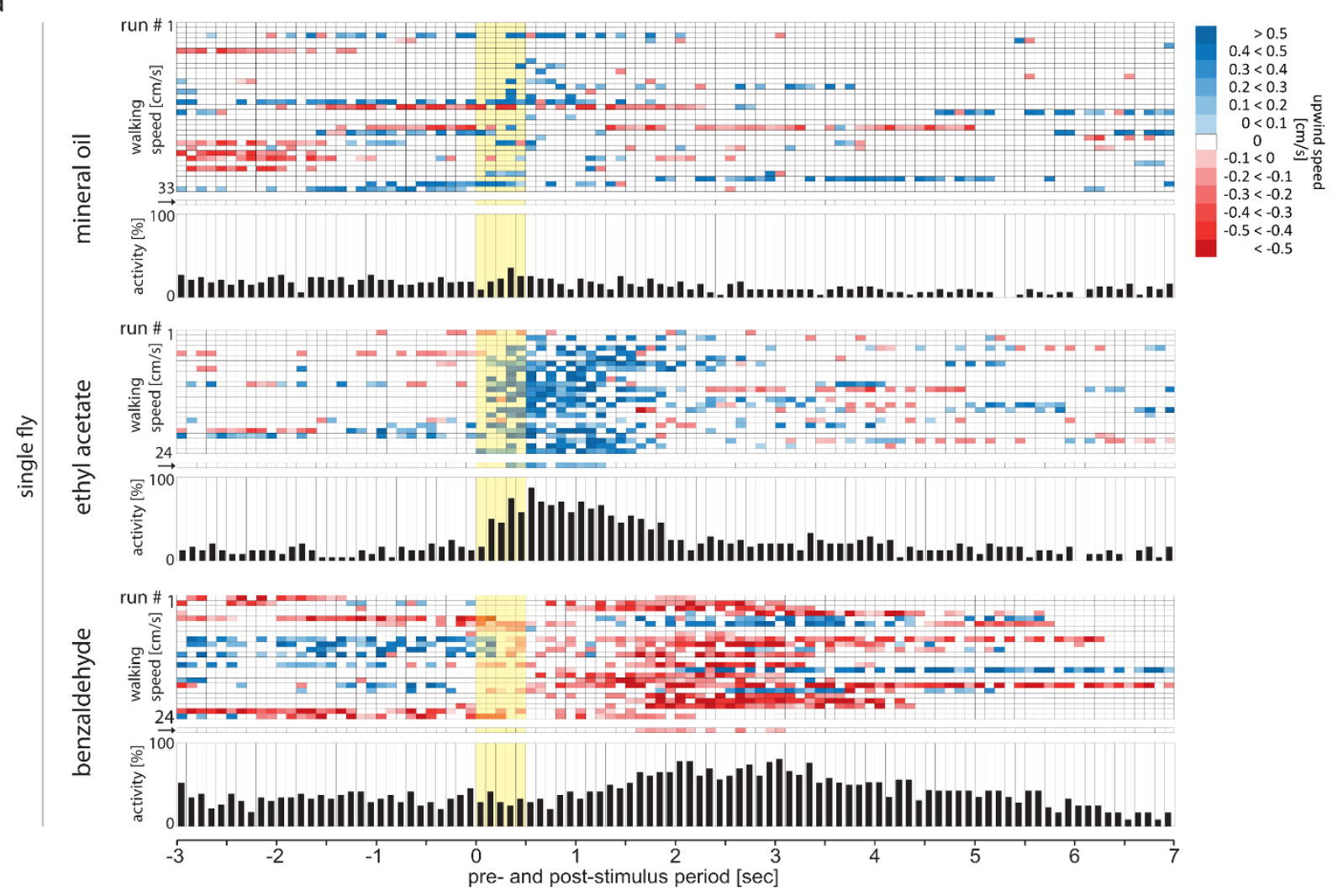

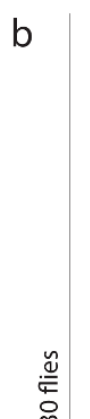
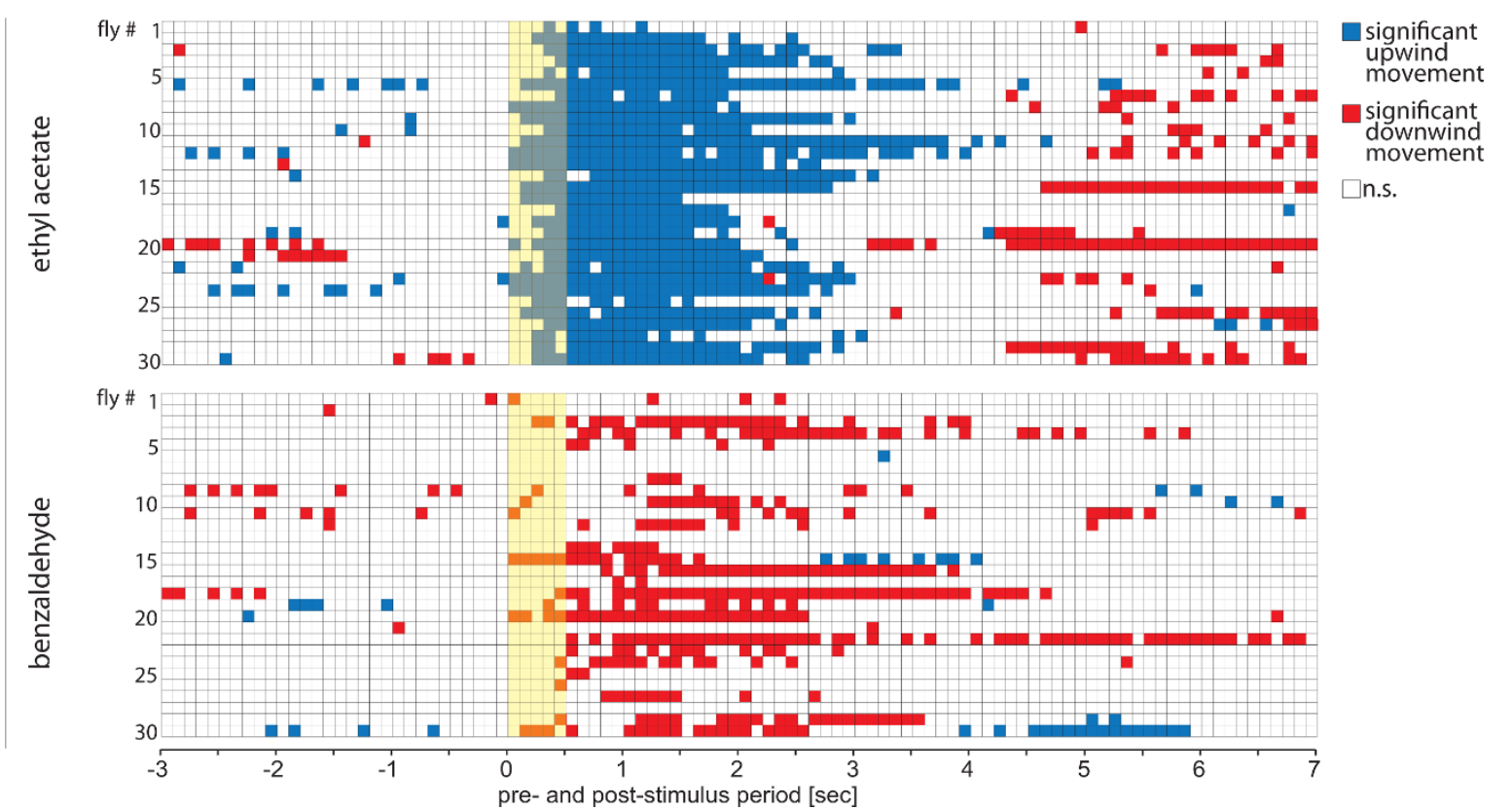

Figure 3 Odor induced responses measured with Flywalk. (a) Ethograms, responses of a single fly to repeated stimuli (duration, $500 \mathrm{~ms}$, onset at 0 s; yellow bar) with mineral oil (as solvent control, $\mathrm{N}=30)$, ethyl acetate $(\mathrm{N}=24)$, and benzaldehyde $(\mathrm{N}=24)$. Each row depicts an individual response to one stimulation over a time period of $10 \mathrm{~s}$ ( $3 \mathrm{~s}$ pre stimulation, $500 \mathrm{~ms}$ stimulation, $6.5 \mathrm{~s}$ post stimulation, temporal resolution, $100 \mathrm{~ms}$ ). Upwind speed is color coded. Arrow marked row, median response during all repeated stimulations with the corresponding odor. Histograms depict activity [\%] per time interval, i.e. proportion of colored squares and total squares per time interval. (b) Statistically analyzed responses of 30 flies to repeated stimulations with ethyl acetate and benzaldehyde. Each row depicts the statistically analyzed response of one individual fly to repeated stimulations with ethyl acetate respectively benzaldehyde, compared to the median response to the solvent. Red square, statistical test reveals that net-responses to odor is smaller than expected value 0 , i.e. fly exhibited significant downwind movement during that 100 -ms time frame; blue square, statistical test reveals that net-responses to odor is larger than expected value 0 , i.e. fly exhibited significant upwind movement during that time frame; white square, statistical test reveals that netresponses to odor does not differ from expected value 0 , i.e. fly did not exhibit significant up- or downwind movement. Yellow area, 500 ms odor stimulus. For statistical analysis see Method section. 

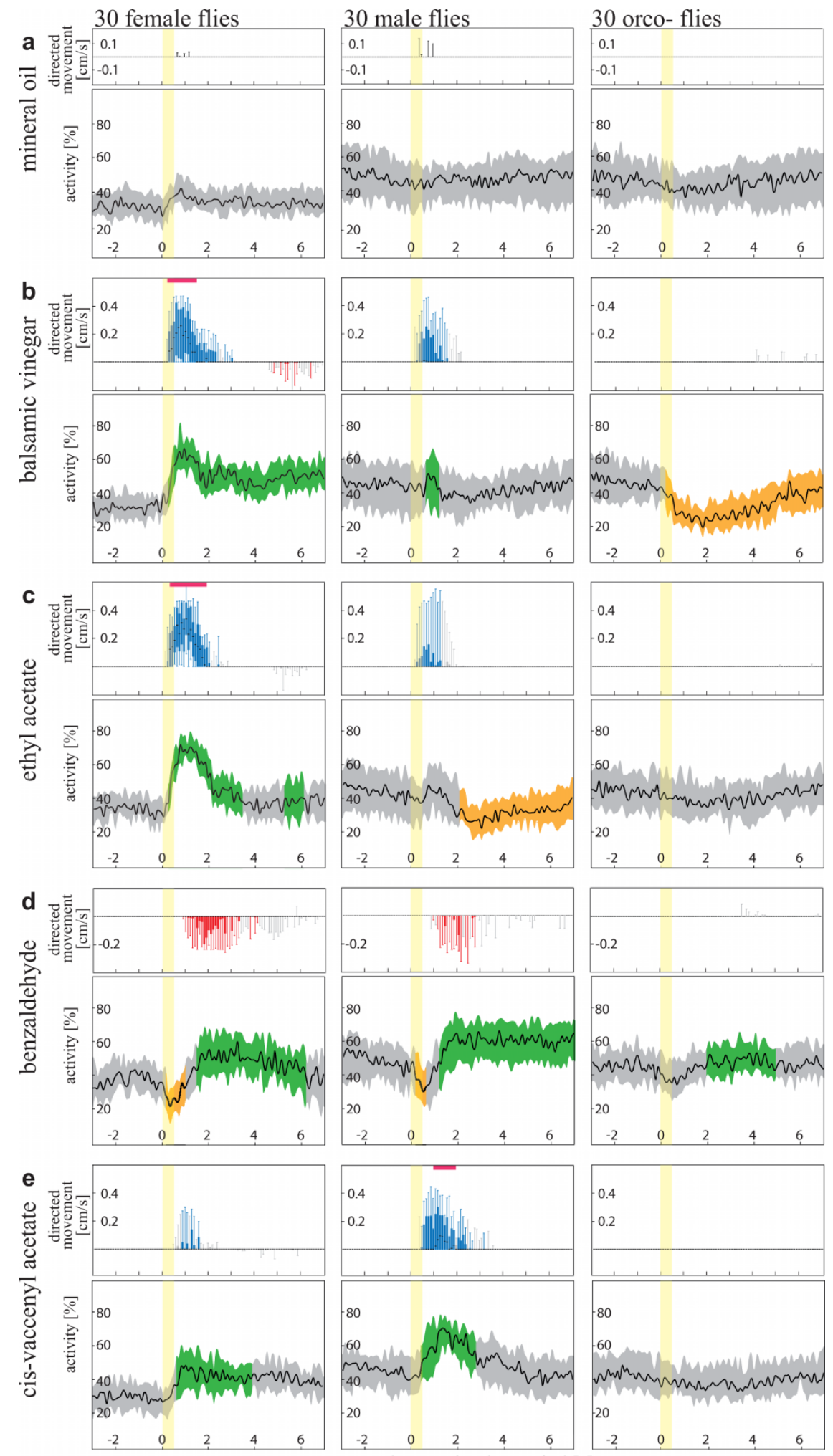

pre- and post-stimulus period $[\mathrm{s}]$

Figure $4 \mid$ Odor induced responses of female and male wildtype Drosophila and female Orco- mutants. (a-e) Tested odors. Top graphs, boxplot representation of odor-induced changes in upwind speed of 30 flies; black line, median upwind speed; box, interquartile range; whiskers, 90 th and 10th percentiles. The blue box-plots depict significantly increased upwind speed compared to the upwind speed during the solvent control situation within the corresponding 100-ms time frame; red box-plots depict significantly decreased upwind speed compared to the upwind speed during the solvent control situation within the corresponding 100-ms time frame, grey box-plots depict no significant difference in upwind speed. Pink colored bar depicts time intervals during which the group showed increased movements compared to the other group (i.e. females vs. males). Low graphs, undirected activity of 30 flies; black line, median activity; shaded area, interquartile range; green, significantly increased activity compared to solvent control; orange, significantly decreased activity compared to solvent control; grey, activities in odor and solvent control situations do not differ. Yellow area, $500 \mathrm{~ms}$ odor stimulus. For statistical analysis see Methods section. 
other hand evoked in Orco - / - flies a delayed increase in activity, as also observed in wildtype flies (Fig. 4d).

As this setup enables us to stimulate individual flies with pulses of volatiles compounds and observe already minute changes in the flies' behavior, we next investigated how flies respond to fly odors, i.e. the headspace of flies (for detailed description of rearing and headspace collection see material section). We recorded the movements of males, virgin females and mated females while they were exposed to pulses containing the headspace of males, virgin females and mated females (Fig. 5). Males, virgin females and mated females responded with higher upwind speeds to the male volatiles compared to the female volatiles (Fig. 5b-d). However, while headspace of mated females evoked no significant response in any of the tested groups, headspace of virgin females was attractive to males. Hence, based on volatile cues flies in general are able to distinguish between sexes and males can evaluate a female's mating status.

\section{Discussion}

Here we present a novel paradigm and instrument for studying the odor-induced behavior of Drosophila. The method features four major advantages over commonly used approaches:

- The positions of 15 individual flies are tracked in parallel.

- Each animal is exposed to an identical air stream carrying identical odor stimuli.

- Stimuli are delivered with high precision in quantity, quality and timing.

- The stimulus delivery system is synchronized in time with the automatic tracking system.

The combination of these requirements allows for

- screening of numerous odors for behavioral relevance,

- in-depth study of odor-evoked behavior of numerous individually tested flies with a so-far unmatched temporal resolution of $100 \mathrm{~ms}$,

- better comparability of behavioral and physiological data (EAGs, calcium imaging, and single sensillum recordings), as both can be obtained with identical olfactory stimuli.

In order to demonstrate the potential of this approach we addressed the following questions:

- Do male and female flies differ in their olfactory responses?

- Do Orco mutant flies respond to olfactory stimuli?

- Are volatile cues sufficient for flies to evaluate sex and mating status of conspecifics?

We found that depending on stimulus quality flies responded with directed runs. Attractive odors (ethyl acetate, balsamic vinegar and cVA) increased the flies' upwind movements, while an aversive odor (benzaldehyde) elicited downwind movements (Fig. 4). Additional information regarding olfactory responses was provided by the evaluation of the flies' undirected activity. While it is evident that increased directed movements also result in increased activity, a decrease in activity, i.e. freezing as a response to odorants (Fig. 4), has so far been overlooked. Furthermore, odor-evoked changes in behavior appear to be consistent during long experimental sessions (Supplementary Fig. S4 online). These findings thus enabled us to evaluate the behavior of individual flies for their olfactory preferences (Fig. 3b, 4, and Supplementary Fig. S5) and sensitivity (Supplementary Fig. S7 online).

We provide evidence that flies exhibit sex-specific responses to food-derived odors (ethyl acetate and balsamic vinegar, Fig. 4b, c) and to a fly-derived pheromone (cVA) (Fig. 4e). The significantly stronger responses of females to food odors might be explained by increased motivation of females. Female flies have higher food requirements due to egg production, and are additionally searching for potential oviposition sites. By contrast, we found no differences between responses of female and male flies to the repellent odors. Upon stimulation with benzaldehyde and 1-octen-3-ol both sexes responded with freezing, which in the case of benzaldehyde was followed by downwind movements (Fig. 4d). Males responded stronger to cVA than females. cVA is involved in different behavioral circuits: after successful mating a male fly transfers $\mathrm{cVA}$ to the female, which reduces her attractiveness to other males ${ }^{23}$. Furthermore cVA has been demonstrated to act as an aggregation pheromone for female and male flies ${ }^{18}$ and - in high concentrations - to induce aggression in males ${ }^{21}$. The observed upwind runs of females thus correspond well with the role of $\mathrm{cVA}$ as an aggregation pheromone. On the other hand, the even stronger responses of males to cVA might be explained by co-acting motivations like aggregation and aggression.

We next asked whether the odor-evoked response of the flies depends on functional olfactory receptors. Based on previous findings showing that mutant flies lacking the olfactory coreceptor Dmel/ Orco are not attracted to a majority of odors, these flies and their larvae have been described as anosmic ${ }^{24}$. We could confirm that Orco flies are not attracted nor repelled by any of the odors tested (Fig. 4). However, by measuring the mutant flies' activity we were able to identify odors (e.g. balsamic acid, benzaldehyde) that caused activity changes. Hence, these volatiles might be detected by ionotropic glutamate-like receptors, another kind of Drosophila chemoreceptors that are not affected by the Orco mutation ${ }^{6,25}$. Again, the analysis of activity offered additional valuable clues about the fly's ability to smell even in the absence of any valence-specific behavior.

The analytic power of the approach became further apparent when we tested flies with headspace collections from males, and virgin and mated females. Producing headspaces of 900 individually selected flies with known sex and mating status is highly labor and time consuming (see Methods). In such a case the advantage of the Flywalk is that it is possible to collect meaningful data during a limited number of experimental sessions (i.e. three experiments already produced data with sufficient statistical power). We found that all female flies responded strongly (i.e. exhibited upwind runs) to the headspace of males (Fig. 5b) but not to the headspace of females (Fig. $5 \mathrm{c}$ and d). So far sex discrimination experiments in Drosophila always included multisensory information like visual, tactile, auditory, gustatory and olfactory cues ${ }^{1,20,26,27}$. Our results show that olfactory cues alone are sufficient for female flies to identify potential mating partners. Like females, males responded to male headspace (Fig. 5b) but not to the one collected from mated females (Fig. 5d), i.e. they discriminated between sexes. Males were however attracted by the virgin female headspace (Fig. $5 \mathrm{c}$ ). This shows that male flies are capable of evaluating the mating status of a potential partner based on olfactory cues. Future experiments using our high-throughput behavioral paradigm - the Flywalk - will be aimed at revealing which volatile components are involved in the evaluation of sex and mating status.

\section{Methods}

Fly rearing. Flies (CantonS, Bloomington) and Orco - /- mutants were maintained at $25^{\circ} \mathrm{C}, 70 \%$ relative humidity under $12 \mathrm{~L}: 12 \mathrm{D}$ in standard food vials

$(25 \mathrm{~mm} \times 95 \mathrm{~mm}$ ) containing standard agar-cornmeal medium. At the age of 4 days flies were starved for 24 hours in a glass vial containing a moist bed of tissue paper. Experiments were conducted with 5-days old flies.

Experimental setup. The setup consisted of 15 glass tubes (length, $18 \mathrm{~cm}, \varnothing$ $0.8 \mathrm{~cm}$, Fig. 1) each containing one fly. The tubes were aligned on an electroluminescent foil (Reichelt, Germany) combined with red plexiglass (allowing light with wave length $>630 \mathrm{~nm}$ to pass). The positions of the flies were recorded with an automatic tracking system (Supplementary Fig. S3 online). As the only light source during the experiment derived from the red illuminating background, any visually induced following behavior of neighboring flies could be excluded. The tubes were connected to an olfactory stimulus generator ${ }^{16}$ that provided continuous air flow (purified compressed house air; temperature, $25^{\circ} \mathrm{C}$; humidity, 75\%; wind speed, $18 \mathrm{~cm} / \mathrm{s}$ ) and - after the flies were allowed to adapt for $30 \mathrm{~min}$ - delivered pulses of up to seven individual odorants and a no-odorant solvent control with a stimulus length of $500 \mathrm{~ms}$ and a stimulus interval of $90 \mathrm{~s}$. 

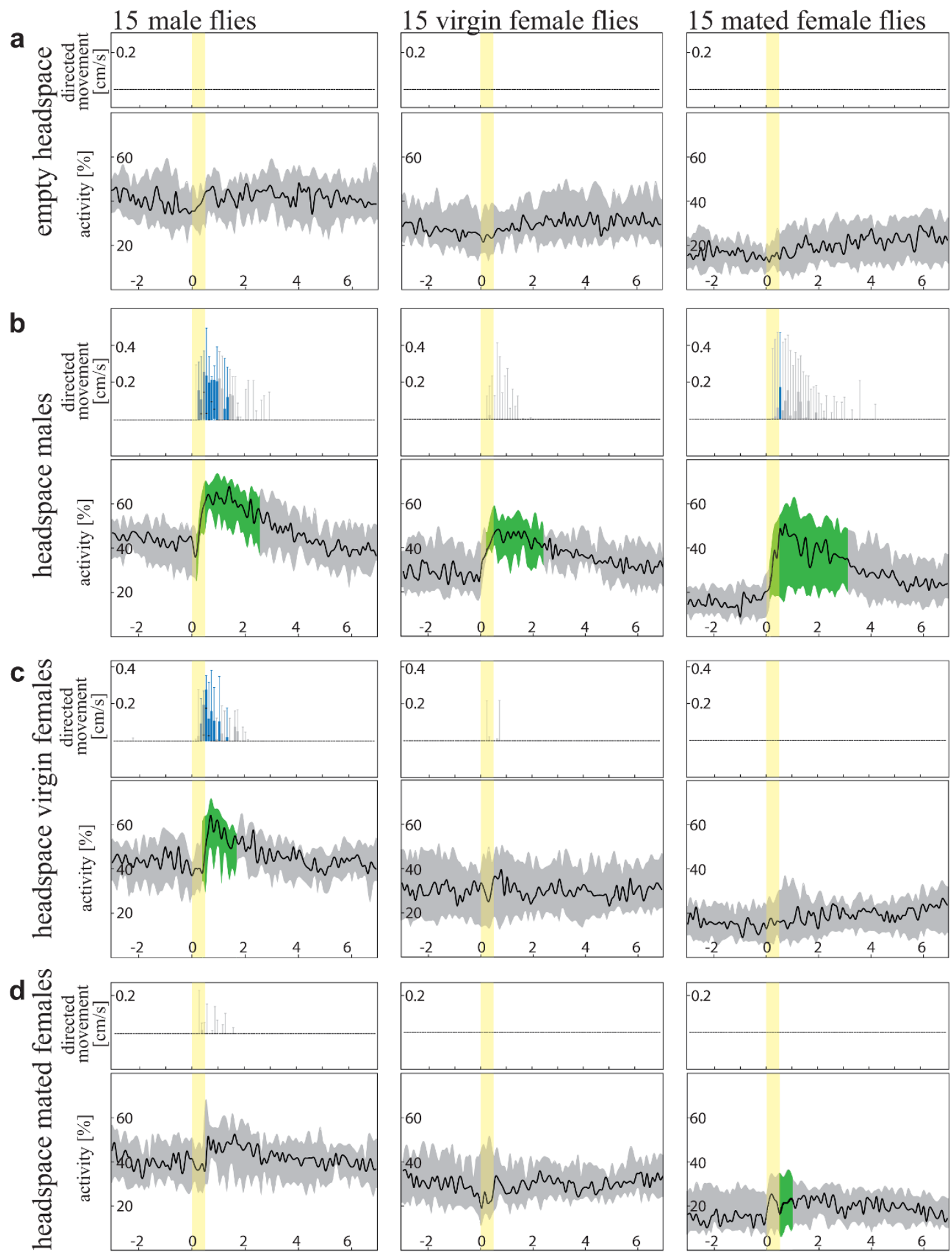

pre- and post-stimulus period [s]

Figure 5 Responses of male, virgin female, and mated female flies to fly odors. (a-d) Tested headspaces of no flies (a), male (b), virgin female (c), and mated female (d) flies. Top graphs, boxplot representation of odor-induced changes in upwind speed of 15 flies; black line, median upwind speed; box, interquartile range; whiskers, 90th and 10th percentiles. The blue box-plots depict significantly increased upwind speed compared to the upwind speed during the solvent control situation within the corresponding 100-ms time frame; red box-plots depict significantly decreased upwind speed compared to the upwind speed during the solvent control situation within the corresponding 100-ms time frame, grey box-plots depict no significant difference in upwind speed. Low graphs, undirected activity of 15 flies; black line, median activity; shaded area, interquartile range; green, significantly increased activity; orange, significantly decreased activity. Yellow area, $500 \mathrm{~ms}$ odor stimulus. For statistical analysis see Methods section. 
(For detailed description of the olfactory stimulation system $\operatorname{see}^{16}$, for a detailed description of the connections between tubes and system see Supplementary Fig. S1 online.)

Stimulus preparation. Each of the seven odor vials contained one odor (either a single odorant, or a complex mixture like balsamic vinegar). Unless otherwise mentioned, odors were dissolved 1:10 in mineral oil. $100 \mu$ lof the dissolved odor were pipetted in a $1 \mathrm{ml}$ Eppendorf tube. One additional vial served as a solvent control. The odor and control vials containing the Eppendorf tubes were sealed hermetically with a stainless steel plug and a rubber O-ring and with corresponding ball-stop check valves at both inlets and outlets.

Preparation of headspace collections from males, and virgin and mated females. For each of the experimental sessions we prepared headspace collections of 100 mated males, 100 virgin females, and 100 mated females. For this purpose, flies were separated by sex at the pupal stage to ensure virginity of female flies. The flies - one group of virgin females and one group of mixed sexes - were kept for three days on standard agar-cornmeal medium. They were then transferred to sugar water for two days to reduce food-derived chemicals sticking to the flies' bodies. Prior to the experiment we equipped the odor vials of the setup with flies, now separated by sex and mating status: one vial with 100 virgin females, one vial with 100 mated females and one vial with 100 mated males. The flies were kept in the vials at room temperature for 5 hours. Afterwards the vials were placed in the freezer $\left(-20^{\circ} \mathrm{C}\right)$ for 10 minutes and the flies were removed. This procedure ensured that the vials now contained volatile compounds derived from the flies. Finally the vials were inserted into the stimulus delivery system, allowed to reach room temperature and were then used for the experiment.

Stimulus characterization. In order to analyze the flies' responses to odor pulses we needed detailed information regarding stimulus characteristics. Therefore, we analyzed pulses within the tubes using a photo ionization device (miniPID, Model 200A, Aurora Scientific Inc.Canada). The miniPID detects and quantifies non-air volatiles with a temporal resolution that resembles that of a fly antenna (Fig. 2a and b). Probing at different positions in the tubes informed us about the speed of the stimuli (Fig. 2c) and ensured that the characteristics of the stimuli regarding stimulus length, concentration, and concentration gradient were consistent at all positions within the tubes (Fig. 2d, for detailed procedure see Supplementary Information online). Digital flowmeters at the end of each hermitically sealed glass tube ensured that the wind speed, i.e. movement of the stimulus, was identical in all tubes.

Data generation and analysis. In order to analyze stimulus-induced responses, freely walking $D$. melanogaster flies were individually tracked (time resolution, ca. $25 \mathrm{~Hz}$, spatial resolution, $0.06 \mathrm{~cm}$, i.e. $25 \%$ of the fly's body length; for detailed description of the tracking system see Supplementary Information). Position coordinates of the flies were synchronized with the timer information of the stimulus delivery system. Based on onset time and speed of the stimulus within the tube and the tracked position of each fly within its tube, a MATLAB routine (The Mathworks, Natick, USA) then calculated the time when the stimulus reached the fly for each individual, i.e. meeting time. In several cases, a fly was outside the region of interest (Fig. 1) when meeting the stimulus and therefore was not included in the evaluation. Thus, one representative experimental session resulted in approximately 22 analyzable runs per fly and compound (Supplementary Fig. S3 online). For each of these runs (which can be assigned unambiguously to a fly and to an odor) the MATLAB routine quantified the linear (i.e. up- and downwind) movements of the fly before, during and after the odor stimulation with a temporal resolution of $100 \mathrm{~ms}$. The ethograms, i.e. the color-coded response matrixes for one fly tested repeatedly with mineral oil, ethyl acetate and benzaldehyde are displayed in Fig. 3a.

\section{Statistical analyzes were conducted to test whether}

- an individual fly increased its up- or downwind movements as a response to pulses of a given odor compared to the control (Fig. 3b and Supplementary Information Fig. S5).

- the sum response (i.e. the summed responses of a group of flies) to an odor differed to the response to the solvent control (Fig. 4 and Supplementary Information Fig. S6)

- the flies' activity changed as a response to pulses of a given odor compared to the control (Fig. 4 and Supplementary Information Fig. S6).

- two groups of flies differed in their responses (i.e. the summed responses of two groups of flies) to the same odor (Fig. 4).

How does an individual fly respond to a given odor? In order to statistically analyze the responses of individual flies to the repeated stimulation with a given odor, we first calculated the median movements before, during, and after stimulation with mineral oil (i.e. the solvent control) for each fly (see median responses in Fig. 3a, single row of the data marked by arrow). We then subtracted the median response to the solvent stimulus (one median response) from each single response to a given odor stimulus (between 7 and 35 responses, compare Supplementary Fig. S3). By this calculation we receive (between 7 and 35) net-responses. If the response to a given odor is the same as to the solvent stimulus one would expect to receive values around 0 for all time intervals before, during and after stimulation. Therefore we use a Wilcoxon-SignedRank-Test to compare for each time interval whether these ( 7 to 35 ) net-values differ from the expected value, i.e. from 0 . If this statistical test reveals that the net-values for a given time interval is larger then 0 , i.e. the heading direction is upwind, the corresponding square appears blue in Figure 3b. If the net-values for a given time interval is smaller then 0 , i.e. the heading direction is downwind, the corresponding square appears red in Figure 3b. If this statistical test reveals that the net-values for a given time interval does not differ from 0 , the corresponding square appears white in Figure $3 \mathrm{~b}$.

When analyzing the summed responses of groups of flies we subtracted the median responses (i.e. 30 median traces) during mineral oil situation (i.e. the solvent control) from the median responses during test situations for each fly. This results in one netresponse per fly and thus, 30 net-responses for the group of 30 tested flies. Assuming that the net-responses would not differ from 0 (because the responses to the solvent and to a given odor were the same) we tested against an expected value using the Wilcoxon signed-rank test. We tested for each time interval whether the 30 net-values differed significantly from the expected value, i.e. from 0 . If this statistical test reveals that the net-values for a given time interval is larger then 0 , i.e. the heading direction is upwind, the corresponding box-and-whisker-plot appears blue in Figures 4 and 5. If the net-values for a given time interval is smaller then 0 , i.e. the heading direction is downwind, the corresponding box-and-whisker-plot appears red in Figures 4 and 5. If this statistical test reveals that the net-values for a given time interval does not differ from 0 , the corresponding box-and-whisker-plot appears grey in Figures 4 and 5.

In addition to the directed movements (i.e. how fast a fly was moving up- or downwind) we quantified the undirected activity of each fly and each time interval (i.e. whether the fly was moving or not, independent from the direction or speed of movement). The activity histograms in Fig. 3a represent examples for a single fly tested with mineral oil (solvent control), ethyl acetate and benzaldehyde. The values of the activity histograms depict the relative proportion of movements for each time frame (i.e. the number of colored squares for each time frame of the ethogram compared to the total number of white and colored squares for each time frame of the ethogram). These activity traces were calculated for each fly, which results in 30 activity traces for each odor. Again, we subtracted the activity traces during control situations from the activity traces during test odor situations for each fly. This results in one net-activity per fly and odor and thus, 30 net-activities for the group of 30 tested flies. Assuming that the net-activity would not differ from 0 (because the activities during the solvent situation and during a given odor situation were the same) we tested against an expected value using the Wilcoxon signed-rank test. We tested for each time interval whether the 30 net-values differed significantly from the expected value, i.e. from 0 . If this statistical test reveals that the net-values for a given time interval is larger then 0 , i.e. the activity during the odor situation is increased compared to the solvent control situation, the corresponding time interval appears green in Figures 4 and 5. If the net-values for a given time interval is smaller then 0, i.e. the activity during the odor situation is decreased compared to the solvent control situation, the corresponding time interval appears orange in Figures 4 and 5. If this statistical test reveals that the net-values for a given time interval does not differ from 0 , the corresponding time interval appears grey in Figures 4 and 5.

To analyze differences between groups tested with the same stimulus we compared the median movements of both groups during each time interval using the Wilcoxon signed-rank test. If the values for a given time interval differ between the groups, the corresponding time intervals are marked with a colored bar in the group showing increased movements in Figure 4.

1. Grosjean, Y. et al. An olfactory receptor for food-derived odours promotes male courtship in Drosophila. Nature 478, 236-U123 (2011).

2. Hallem, E. A. \& Carlson, J. R. Coding of odors by a receptor repertoire. Cell 125, 143-160 (2006).

3. Bhandawat, V., Olsen, S. R., Gouwens, N. W., Schlief, M. L. \& Wilson, R. I. Sensory processing in the Drosophila antennal lobe increases reliability and separability of ensemble odor representations. Nat. Neurosci. 10, 1474-1482 (2007).

4. Sachse, S. et al. Activity-dependent plasticity in an olfactory circuit. Neuron $\mathbf{5 6}$ 838-850 (2007).

5. Semmelhack, J. L. \& Wang, J. W. Select Drosophila glomeruli mediate innate olfactory attraction and aversion. Nature 459, 218-U100 (2009).

6. Silbering, A. F. et al. Complementary function and integrated wiring of the evolutionarily distinct Drosophila olfactory subsystems. J. Neurosci. 31, 1335713375 (2011).

7. Ruebenbauer, A., Schlyter, F., Hansson, B. S., Lofstedt, C. \& Larsson, M. C. Genetic variability and robustness of host odor preference in Drosophila melanogaster. Curr. Biol. 18, 1438-1443 (2008).

8. Tully, T. \& Quinn, W. G. Classical-conditioning and retention in normal and mutant Drosophila melanogaster. J. Comp. Physiol., A 157, 263-277 (1985).

9. Faucher, C., Forstreuter, M., Hilker, M. \& de Bruyne, M. Behavioral responses of Drosophila to biogenic levels of carbon dioxide depend on life-stage, sex and olfactory context. J. Exp. Biol. 209, 2739-2748 (2006).

10. Claridge-Chang, A. et al. Writing Memories with Light-Addressable Reinforcement Circuitry. Cell 139, 405-415 (2009).

11. Keller, A., Zhuang, H. Y., Chi, Q. Y., Vosshall, L. B. \& Matsunami, H. Genetic variation in a human odorant receptor alters odour perception. Nature 449, 468U6 (2007).

12. Budick, S. A. \& Dickinson, M. H. Free-flight responses of Drosophila melanogaster to attractive odors. J. Exp. Biol. 209, 3001-3017 (2006). 
13. Bhandawat, V., Maimon, G., Dickinson, M. H. \& Wilson, R. I. Olfactory modulation of flight in Drosophila is sensitive, selective and rapid (vol 213, pg 3625, 2010). J. Exp. Biol. 213, 4313-4313 (2010).

14. Duistermars, B. J., Chow, D. M. \& Frye, M. A. Flies require bilateral sensory input to track odor gradients in flight. Curr. Biol. 19, 1301-1307 (2009).

15. Lehmann, F. O. \& Dickinson, M. H. The changes in power requirements and muscle efficiency during elevated force production in the fruit fly Drosophila melanogaster. J. Exp. Biol. 200, 1133-1143 (1997).

16. Olsson, S. B. et al. A novel multicomponent stimulus device for use in olfactory experiments. J. Neurosci. Methods 195, 1-9 (2011).

17. Kwon, Y. et al. Drosophila TRPA1 channel is required to avoid the naturally occurring insect repellent citronellal. Curr. Biol. 20, 1672-1678 (2010).

18. Bartelt, R. J., Schaner, A. M. \& Jackson, L. L. Cis-vaccenyl acetate as an aggregation pheromone in Drosophila melanogaster. J. Chem. Ecol. 11, 1747-1756 (1985)

19. Benton, R. Sensitivity and specificity in Drosophila pheromone perception. Trends Neurosci. 30, 512-519 (2007).

20. Kurtovic, A., Widmer, A. \& Dickson, B. J. A single class of olfactory neurons mediates behavioural responses to a Drosophila sex pheromone. Nature 446, 542 546 (2007).

21. Wang, L. \& Anderson, D. J. Identification of an aggression-promoting pheromone and its receptor neurons in Drosophila. Nature 463, 227-U114 (2010).

22. Knaden, M. (submitted).

23. Zawistowski, S. \& Richmond, R. C. Inhibition of courtship and mating of Drosophila melanogaster by the male-produced lipid, cis-vaccenyl acetate. J. Insect Physiol. 32, 189-192 (1986).

24. Larsson, M. C. et al. Or83b encodes a broadly expressed odorant receptor essential for Drosophila olfaction. Neuron 43, 703-714 (2004).

25. Benton, R., Vannice, K. S., Gomez-Diaz, C. \& Vosshall, L. B. Variant ionotropic glutamate receptors as chemosensory receptors in Drosophila. Cell 136, 149-162 (2009).
26. Murthy, M. Unraveling the auditory system of Drosophila. Curr. Opin. Neurobiol. 20, 281-287 (2010).

27. Everaerts, C., Farine, J.-P., Cobb, M. \& Ferveur, J.-F. Drosophila cuticular hydrocarbons revisited: Mating status alters cuticular profiles. Plos One 5 (2010).

\section{Acknowledgments}

This study was supported by fundings from the Max Planck Society to BSH.

\section{Author contribution}

KS performed the experiments. KS, DV, and MK have constructed the behavioral paradigm. KS, $\mathrm{SB}, \mathrm{ZM}, \mathrm{BSH}$, and MK have written the manuscript. SB, ZM, and PV have written the software and have edited the manuscript. RG has written additional routines to analyze the data.

\section{Additional information}

Supplementary information accompanies this paper at http://www.nature.com/ scientificreports

Competing financial interests: I declare that the authors have no competing interests as defined by Nature Publishing Group, or other interests that might be perceived to influence the results and/or discussion reported in this manuscript.

License: This work is licensed under a Creative Commons

Attribution-NonCommercial-ShareAlike 3.0 Unported License. To view a copy of this license, visit http://creativecommons.org/licenses/by-nc-sa/3.0/

How to cite this article: Steck, K. et al. A high-throughput behavioral paradigm for Drosophila olfaction - The Flywalk. Sci. Rep. 2, 361; DOI:10.1038/srep00361 (2012). 
SUBJECT AREAS: ANIMAL BEHAVIOUR

NEUROSCIENCE BIOLOGICAL MODELS OLFACTORY SYSTEM SCIENTIFIC REPORTS: $2: 361$ DOI: $10.1038 /$ srep00361

Published: 12 April 2012

Updated:

12 March 2013
ERRATUM: A high-throughput behavioral paradigm for Drosophila olfaction - The Flywalk

Kathrin Steck' ', Daniel Veit' , Ronald Grandy' , Sergi Bermúdez i. Badia' ${ }^{2}$ Zenon Mathews ${ }^{3}$, Paul Verschure ${ }^{3}$, Bill S. Hansson' \& Markus Knaden'

\footnotetext{
${ }^{1}$ Max Planck Institute for Chemical Ecology, Jena, Germany, ${ }^{2}$ Madeira Interactive Technologies Institute, University of Madeira Carnegie Mellon j Portugal - Madeira Tecnopolo, 9020-105 Funchal, Portugal, ${ }^{3}$ Laboratory of Synthetic Perceptive, Emotive and Cognitive Systems, ICREA-UPF, Barcelona, Spain.
}

The original version of this Article contained a typographical error in the spelling of the author Sergi Bermúdez $i$ Badia which was incorrectly given as Sergi Bermdez i Badia. Furthermore, the affiliation for Paul Verschure and Zenon Mathews was incorrectly given as, Institute of Audio-Visual Studies, University Pompeu Fabra, Barcelona, Spain rather than, Laboratory of Synthetic Perceptive, Emotive and Cognitive Systems, ICREA-UPF, Barcelona, Spain. Both these errors have now been corrected in the Article. 